Cita bibliográfica: Luque Martínez, T., Doña Toledo, L. y Faraoni, N. (2020). Turismo y gestión de marketing. Análisis comparativo entre parques y estaciones de esquí. Investigaciones Turísticas (20), pp. 143-171. https://doi. org/10.14198/INTURI2020.20.07

\title{
Turismo y gestión de marketing. Análisis comparativo entre parques y estaciones de esquí
}

Tourism and marketing management. Comparative analysis between parks and ski stations

Teodoro Luque Martínez (iD, Universidad de Granada, España

tluque@ugr.es

Luis Doña Toledo iD, Universidad de Granada, España

luisdt@ugr.es

Nina Faraoni iD, Universidad de Granada, España

ninaf@ugr.es

\section{RESUMEN}

El turismo de montaña, que incluye estaciones de esquí y áreas naturales protegidas, ha de buscar un equilibrio entre satisfacer las necesidades de los visitantes y el respeto al medio ambiente. En dicha búsqueda, el marketing puede desempeñar un importante papel. Este trabajo trata de responder a la falta de estudios sobre la aplicación del marketing en las actividades de turismo de montaña y, en particular, en la comunicación en redes sociales comparando la aplicación y la percepción que se tiene del marketing en las estaciones de esquí con los parques naturales. Para ello, se ha realizado una auditoría de marketing preguntando a directivos y técnicos de unos y otros, y se han analizado 26 indicadores de redes sociales (Twitter y Facebook). Las estaciones de esquí presentan un mayor enfoque al marketing salvo en aspectos relativos al impacto ambiental. Los resultados muestran la diferente sensibilidad y los aspectos donde hay mayor divergencia, distinguiendo por el grado de importancia como por el grado de adecuación que se les otorga mediante la matriz de importancia-adecuación. También es mayor el dinamismo en redes sociales de las estaciones de esquí.

Palabras clave: Turismo de montaña; áreas naturales protegidas y marketing; estaciones de esquí y marketing; redes sociales y parques nacionales-naturales; redes sociales y estaciones de esquí. 


\section{ABSTRACT}

Mountain tourism, which includes ski resorts and protected natural areas, has to strike a balance between meeting the needs of visitors and respecting the environment. In the search for this balance, marketing can play an important role. This paper seeks to respond to the lack of studies on the application of marketing in mountain tourism activities and, in particular, in communication in social networks by comparing the application and the perception of marketing in ski resorts with that of natural and national parks. To this end, a marketing audit was carried out, based on interviews with managers and technicians of each and analyzing 26 indicators of social networks (Twitter and Facebook). The ski resorts have a greater focus on marketing except in aspects related to environmental impact. The results show the different levels of sensitivity and those aspects where there is greater divergence, distinguishing the degree of importance and degree of adequacy that is granted by the importance-adequacy matrix. There is also greater dynamism in the social networks of ski resorts.

Keywords: Mountain tourism; natural protected areas and marketing; ski resorts and marketing; social networks and national-natural parks; social networks and ski resorts.

\section{INTRODUCCIÓN}

La biodiversidad mundial continúa en declive a un ritmo alarmante. Con la Conferencia de París de 2015 se establecieron algunos objetivos fundamentales, entre los cuales resaltaba el de reducir la emisión de gases invernadero y asegurar la integridad de los ecosistemas a través de educación, participación pública y cooperación. El crecimiento del turismo ha contribuido al aumento de estos dos problemas, al mismo tiempo que ha llegado a ser una de las actividades económicas más importantes del mundo (Hall, O’Mahony y Gayler, 2017). Una parte creciente de esta actividad tiene lugar en las montañas a través de parques (con diferente grado de protección según su categoría) y de estaciones de esquí.

Las áreas naturales protegidas, que incluyen los parques nacionales/naturales en muchos casos de montaña (en adelante PN) son la base de la conservación de la biodiversidad, con importantes implicaciones sociales y económicas, además de las medioambientales en el territorio de influencia.

El $14,8 \%$ de la superficie total del planeta es actualmente un área protegida (UICN, 2016). La creciente superficie protegida del planeta requiere de estructuras organizativas que puedan adoptar formas y estrategias muy diferentes. Es pertinente preguntarse por la adecuación del funcionamiento de estas estructuras organizativas, y un aspecto importante lo constituye la gestión de marketing de dichas áreas (Canadian Heritage Canada Parks, 2017). La planificación realizada por las autoridades encargadas de la gestión de áreas naturales protegidas ha reflejado el deseo de incorporar programas de marketing estratégico dentro de estos entornos (SEMARTAT-CONANP 2014; MAPAMA 2016; NPS-US 2017). Las principales motivaciones para la implementación de planes de marketing son: la preocupación por alcanzar las metas propuestas, el interés creciente por medir la satisfacción de los visitantes y la necesidad de atraer nuevas visitas, además de reconocer la necesidad de establecer estrategias 
enfocadas a las conductas del mercado y, de esta manera, desarrollar actividades con fines recreativos y turísticos (Jamrozy, 2007).

Por otro lado, las estaciones de esquí tienen un impacto económico y social relevante, pues dinamizan y favorecen el desarrollo de las zonas de influencia, bien como actividad principal, bien como actividad complementaria a otras (Benson et al., 2013, Kruger et al., 2017). Más de 80 países de todo el mundo ofrecen deportes de invierno, cuya actividad supone en torno al $5 \%$ de turismo mundial. Por su importancia económica, estos destinos turísticos deben estudiar el mercado y su entorno, existiendo la necesidad de la incorporación del marketing a su gestión. Por estas razones, cabe preguntarse si esto realmente se está produciendo. En la literatura se puede encontrar que, desde la perspectiva de marketing, las investigaciones existentes se centran en los estudios de los atributos, de la calidad de servicio y de la valoración por parte de los usuarios, especialmente esquiadores.

Las auditorías de marketing suponen una herramienta fundamental para mejorar estos servicios. Es un instrumento de evaluación minuciosa, sistemática y periódica de las metas, estrategias, organización y rendimiento del grupo de mercadotecnia (McDonald y Wilson, 2016). Lo más importante es que es una visión del pasado y presente para afrontar exitosamente el futuro (Lamb y Crompton, 2017).

En la actualidad, dentro de las estrategias de marketing, tiene un papel esencial el empleo de redes sociales que pueden contribuir a mejorar la relación con los distintos stakeholders, y a conocerlos mejor (Alarcón et al. 2018). Las redes sociales como Facebook o Twitter forman, actualmente, una parte importante de la vida diaria de las personas, trasladando sus opiniones y formando la imagen de las empresas (Alalwan et al., 2017). Por lo tanto, las redes sociales se han observado como una de las herramientas más eficientes e influyentes para concienciar y crear identidad con todo tipo de organizaciones (sociales, deportivas, comerciales, educativas o relacionadas con el medio ambiente) (Algharabat et al., 2017; Zhu y Chen, 2015). Las redes sociales han cobrado una especial importancia, sin embargo, su influencia y análisis no se ha realizado en actividades relacionadas con la montaña (Rothschild, 2011).

A pesar de la importancia tanto de las estaciones de esquí como de los parques, no se han identificado investigaciones que evalúen la aplicación integral del marketing en ellos, en definitiva, que realicen una auditoría y comparen para ambos el grado de importancia y de adecuación de las aplicaciones del marketing o sobre sus principales dimensiones: entorno, estrategia, organización, sistemas, productividad y funciones (Kotler y Dubois 1986). Del mismo modo, tal como indican Filo et al. (2015), a pesar de que hay un buen número de estudios que han examinado las redes sociales en empresas comerciales, hay ciertos contextos o áreas (como el sector de deportes de invierno) donde es necesario investigar el uso, el impacto y las dimensiones teóricas de las redes sociales.

En este contexto, el objetivo principal de este trabajo es hacer un análisis comparado de la gestión de marketing en estaciones de esquí y PN, desde la perspectiva de sus dirigentes, comparando sus actividades con una especial atención a las redes sociales. De forma concreta, los objetivos son: 
- Realizar una auditoría de marketing para identificar las principales fortalezas y debilidades de la gestión de las estaciones de esquí y parques naturales.

- Comparar las diferencias en la aplicación del marketing entre organizaciones de esquí y áreas naturales protegidas.

- Describir los comportamientos en las redes sociales (Twitter y Facebook) y comprobar si hay diferencias entre estaciones de esquí y áreas naturales protegidas.

Se persigue contribuir a la literatura dada la escasa investigación acerca de estos tipos de sectores a través de una metodología cuantitativa, mediante auditorías internas y métricas de Twitter y Facebook.

\section{PARQUES NACIONALES/NATURALES (PN), ESTACIONES DE ESQUÍ Y GESTIÓN DE MARKETING}

La literatura académica sobre auditoría de marketing (Kotler y Dubois, 1986) en servicios o sectores relacionados con esquí y parques es prácticamente inexistente. Sin embargo, la variedad y amplitud de la oferta de actividades en la montaña y en áreas protegidas en general, exige análisis y planificación de marketing del destino. Más aún si se considera el punto de vista del visitante, en la gestión de estos destinos es necesario un uso mayor de herramientas de marketing (Pearce y Schänzel, 2013). La importancia alcanzada por estos destinos aconseja conocer el comportamiento, las actitudes determinadas, por ejemplo, por la emoción que provocan (Kang et al., 2011) o las preferencias de los usuarios y sus consecuencias sobre el entorno. Para ello, es fundamental la realización de auditorías de marketing internas.

No existe consenso sobre la realización y concepto de la auditoría de marketing, los auditores desarrollan normalmente sus propios métodos específicos, en función de las circunstancias o utilizando los métodos más accesibles (Thaghian y Shaw, 2008). Desde un punto de vista conceptual, según Kotler y Keller (2009), la auditoría de marketing es un examen exhaustivo e independiente del entorno y la actividad de la empresa con el fin de determinar las áreas y oportunidades problemáticas y recomendar un plan de acción para mejorar el marketing de la empresa. Wilson (2005) define la auditoría de marketing como un método autoadministrado para identificar y lograr el uso de recursos de marketing. Según Mokwa (1986), la auditoría de marketing puede verse como una política de innovación utilizada primero para aumentar la conciencia y la comprensión, y luego para implementar y evaluar los métodos y el rendimiento de marketing, siendo un instrumento de intervención y un agente de cambio. Da Gama (2012) lo considera como una herramienta imprescindible, pero olvidada por los gestores de marketing.

Kotler (1993), especifica que las auditorías deben caracterizarse por cuatro aspectos: (1) comprensivas (deben cubrir todas las principales actividades de marketing de una empresa y no sólo sus aspectos problemáticos); (2) sistemáticas (involucrar una secuencia ordenada del diagnóstico del mercado de la organización, el sistema interno y las actividades específicas); (3) independientes (la auditoría de marketing corre a cargo generalmente de un consultor externo o un miembro interno que tenga experiencia, credibilidad e independencia 
suficientes); (4) periódicas (deben repetirse cada tiempo para conocer la evolución de la aplicación de marketing).

En el proceso de auditoría de marketing, las empresas utilizan principalmente métodos cualitativos (cuestionario y análisis de datos de ventas y empresariales), y solo unos pocos cuantitativos (Cheng, 2010). El enfoque para la auditoría de marketing consiste en utilizar una lista de verificación o evaluación de preguntas de diagnóstico (da Gama, 2012). La respuesta a estas preguntas proporcionará información sobre la "salud" de marketing de la organización.

En el marketing turístico alcanza una importancia notable el hecho de que estas organizaciones están orientadas hacia un marketing sostenible, por lo que la auditoría de marketing consiste en resaltar el desempeño de las acciones emprendidas, tanto en términos de organización de ganancias, como especialmente por la imagen entre los consumidores y las partes interesadas (Serbanica et al., 2015). Después de identificar oportunidades y amenazas en el mercado, debido a los factores influyentes en el micro y macro entorno, una empresa establecerá la estrategia general de desarrollo y la estrategia de marketing. El punto de partida está representado por la actividad de marketing en toda la empresa (Morgan, Clark y Gooner, 2002).

Referido específicamente a las estaciones de esquí, la gestión de marketing es un eje fundamental en sus estrategias debido a su necesidad de atraer visitantes y desempeñar sus servicios en entornos altamente competitivos. En este caso, en la literatura predominan los estudios que toman en consideración la perspectiva del esquiador, en particular, el interés sobre los principales atributos que debe tener una estación de esquí de alta calidad y su valoración (Hall, O'Mahony y Gayler, 2017) así como los factores de elección de una determinada estación (Mätzler y Siller, 2003; Dickson y Faulks, 2007) o que determinan el perfil demo y psicográfico de los esquiadores (Konu et al., 2011). Según Hall et al. (2017) la diversión, el transporte, los empleados y el alojamiento son los factores más importantes de la satisfacción de los esquiadores. Miragaia et al. (2016) identifican cinco factores de satisfacción: instalaciones y equipos, atributos de las montañas, servicios de resort, restaurantes, alojamiento y actividades sociales y acceso, mientras que Godfrey (1999) los resume en condiciones de nieve y variedad de las pendientes.

Son pocos los estudios que han analizado la opinión de otros agentes dentro de las estaciones como directivos, empleados u profesionales del marketing. Como aproximación a esto, Ismert y Petrick (2004) analizaron la cultura organizacional de las estaciones, concluyendo que los empleados valoraban la socialización (relación con los compañeros, y desempeño y trato de los directivos) como lo más importante para lograr un correcto desempeño de la empresa. Tjørve, Lien y Flognfeldt (2015) encontraron que las políticas de marketing tenían escasa repercusión entre los esquiadores.

Hudson y Shephard (1998) y Hwa-Ryong y Sung-Kyeom (2010) analizaron las estaciones de esquí a través de la metodología de matriz importancia-adecuación (IPA). Los esquiadores otorgaban una mayor importancia a los servicios de información, alojamiento, tiendas de esquí, y pistas de esquí y remontes fundamentalmente. Matzler y Siller (2003) aplicaron una 
variación denominada como matriz de importancia-percepción en la cual establecían las principales motivaciones de los esquiadores.

Mientras que en las estaciones de esquí se ha dado una mayor importancia a la percepción del turista para atraerlo e incrementar los beneficios, en el caso de los PN el reto es conciliar su atractivo como destino, proporcionando una agradable experiencia al visitante junto con un desarrollo sostenible para su entorno, es decir, sin comprometer sus recursos naturales (Goeldner y Ritchie, 2012; Pérez y Ramírez, 2013). Los directivos de los PN se encuentran constantemente ante el dilema de gestionar el espacio y su uso por las personas, a la vez que han de preservar la integridad de la naturaleza, cultura y tradiciones que caracterizan la zona (Gilmore y Simmons, 2007; Lai et al., 2009).

Para una gestión eficaz, los directivos de los PN deben saber cuantificar los beneficios que el parque proporciona a sus visitantes, como pueden ser la diversión y el tiempo libre o la continuidad de la biodiversidad existente en ellos (Benson et al., 2013). La mayoría de los directivos están preparados en cuestiones medioambientales, pero no tanto sobre turismo o comercialización (Kihima, 2014).

Desde una perspectiva de marketing como disciplina que estudia los comportamientos en el intercambio y sus consecuencias para la sociedad y el entorno, por tanto, perspectiva de macromarketing (Hunt, 1983; Layton y Grossbart, 2006), la gestión de los PN no puede estar desligada del concepto de turismo sostenible, ni del marketing y sus técnicas, que impactan directamente o indirectamente sobre las comunidades locales y su economía (Sharpley y Pearce, 2007; Olcina, 2012). Sin embargo, apenas se ha prestado atención a cómo las empresas o entidades que intervienen y gestionan los espacios naturales han desarrollado o adaptado sus estrategias de marketing, ni tampoco a cuál es la visión de directivos o empleados sobre la práctica de tales estrategias. La gestión de marketing de los PN puede contribuir con diversos beneficios como la disminución de los costes de gestión (Corbera et al., 2007) o la creación de la marca del destino natural (Reinius y Fredman, 2007; Kihima, 2014). En definitiva, cuanto mejor gestionados con unos valores de preservación claros, mejor se enfrentarán las amenazas a las que están sometidos.

\section{PARQUES NACIONALES/NATURALES (PN), ESTACIONES DE ESQUÍ Y REDES SOCIALES}

Las comunicaciones se ven facilitadas por la llegada de las tecnologías y, en particular, de las redes sociales, que han supuesto un enorme cambio en la actuación por parte de las organizaciones (Järvinen et al., 2012). Una red social es un grupo interconectado de personas (Doyle, 2007), y está compuesta por siete recursos funcionales: identidad, conversaciones, compartición, presencia, relaciones, reputación y grupos (Kietzmann et al., 2011). Dichas redes tienen un gran poder de influencia, son una vía para establecer diálogos y mantener contacto con los públicos objetivo, hecho que cada vez tiene mayor importancia en las organizaciones (Daries-Ramon et al., 2016).

Hoy en día, las redes sociales son un buen predictor de las preferencias de los consumidores (Gecti y Dastan, 2013; Paniagua y Sapena, 2014), y aportan beneficios a las empresas, sobre todo debido a los efectos del boca-oído (Bulearca y Bulearca, 2010) que es una de las 
herramientas de marketing más potente, además, de ser fácil de observar e investigar (Hung y Li, 2007; Kim et al., 2015). Gracias a todo el intercambio de información que se produce en las redes sociales, se puede crear contenido a partir de los mismos usuarios, reuniendo información sobre características, comportamientos y preferencias (Castronovo y Huang, 2012). Las redes sociales están siendo reconocidas como una herramienta, y los gerentes abogan por su inclusión en la estrategia de marketing ya que los clientes, a menudo, confían en estas plataformas para interactuar con amigos, marcas u organizaciones (Rapp et al., 2013).

Dentro del marketing deportivo, donde se enmarca el esquí o la montaña, se han demostrado los beneficios del uso de redes sociales. Por ejemplo, Baena (2016) mostró que los sitios web tradicionales de los equipos de futbol ya no son suficientes, ya que las redes sociales, como medio interactivo, favorecen la elección y el compromiso con la marca o empresa. loakimidis (2010), analizando cuentas de profesionales deportivos, mostraba la mejora de las relaciones con sus seguidores.

Sin embargo, muchas empresas tienen una deficiente puesta en marcha por variados motivos: estrategia no muy bien definida; mensajes poco consistentes (Rapp et al., 2013); percepción que tienen los directivos acerca de estos instrumentos tecnológicos (Zaglia, 2013); o sensación de falta de control por parte de los gestores (Järvinen et al., 2012).

Las relaciones con los consumidores se ven beneficiadas (Castronovo y Huang, 2012) mediante las redes sociales, ya que ayudan a la creación de relaciones duraderas y basadas en la confianza a través de la implicación, colaboración e interacción empresa/usuario (Rapp et al., 2013). Las principales funciones de las redes sociales son interactuar con los clientes y facilitar la interacción entre clientes/usuarios sin la intervención de la empresa (Alarcón et al., 2018). Este intercambio de conocimiento que se genera a través de las comunicaciones virtuales crea más conocimiento que, con el tiempo, se transforma en una ventaja para la empresa (Hung y Li, 2007).

Para crear estas relaciones fuertes con los seguidores hay que crear contenidos que despierten las emociones de los consumidores, para que se sientan identificados con la marca a través de imágenes, símbolos y videos (Hudson et al., 2015). La "telepresencia" (considerada como la capacidad de la página de la red social de transportar psicológicamente el usuario dentro del mundo creado por la organización), y la "presencia social" (la capacidad del medio de comunicación de hacer sentir el usuario psicológicamente comprometido con otros usuarios) aumentan la implicación general de los usuarios con la organización, y su efectividad depende de la capacidad del organismo de simular una experiencia directa para el interlocutor (Algharabat et al., 2018), esto puede ser especialmente relevante para los PN.

A pesar de la abundancia de literatura sobre redes sociales, son escasas las investigaciones que trataran la relación entre redes sociales y desempeño (Paniagua y Sapena, 2014). Según Rothschild (2011) los gestores de los lugares de deporte y de entretenimiento, como las estaciones de esquí y los PN, en su mayoría, utilizan las redes sociales como parte fundamental de su estrategia, aparte de recibir más beneficios comparados con aquellos que no las utilizan.

Algunas investigaciones ponen de manifiesto que las redes sociales afectan el desempeño de la empresa a través de cuatro canales: capital social (la manera en que los medios 
sociales afectan a las relaciones de la empresa con la sociedad); preferencias reveladas (medida en que las redes sociales revelan las preferencias de los consumidores); marketing (medida en que el marketing social, compuesto por interacciones, "compartir", likes, etc. se transforma en capacidades financieras, por ejemplo en ventas); y redes corporativas (medida en la que los recursos corporativos, como los grupos y la relaciones con los stakeholders, se transforman en capacidades de rendimiento) (Paniagua y Sapena, 2014).

Un aspecto fundamental a la hora de analizar las redes sociales son las propias métricas que se emplean para medir el desempeño. La literatura sobre cómo medir el éxito en redes sociales ha crecido en los últimos años (Coelho et al., 2016). La investigación centrada en las redes sociales ha producido resultados considerables sobre la interacción y la dinámica entre las personas y las organizaciones, pero aún se encuentra en sus primeras etapas, ya que ni siquiera el término redes sociales tiene una definición universalmente aceptada, y no existe una tipología estándar de redes sociales, así como de sus métricas (Weller, 2015).

Existen numerosas métricas de redes sociales y existe una controversia con respecto a la elección del uso de indicadores apropiados (Peters et al., 2013). Un aspecto común para la investigación en este campo es utilizar los datos de las redes sociales para comprender la variación de las variables no económicas, como los gustos, comentarios y acciones (Luarn et al., 2015). Las métricas son necesarias para justificar lo que se está llevando a cabo ante la organización y si esto está teniendo éxito.

Después de crear perfiles para comunicarse con los consumidores, las empresas y las marcas incorporan contenidos como noticias, fotos y videos, buscando elevar los niveles de visitantes y las métricas. Algunos estudios analizan la cantidad de elementos (texto, foto o video) y el supuesto impacto positivo en las respuestas de los consumidores como comentarios, "me gusta" y "compartir" (Kim et al., 2015). Otro grupo de estudios se centra en clasificar los tipos de publicaciones, la riqueza de contenido y cómo las imágenes o videos producen una influencia positiva en los "me gusta" y los comentarios. Un último grupo se centra en la conexión emocional entre el contenido de la red social y la marca u organización (Sabate et al., 2014).

Las cinco métricas más importantes para Twitter y Facebook son según Coelho et al. (2016): contenido (videos, publicaciones, fotos); comentarios (a partir de post o creación de eventos); números de "me gusta" y seguidores; respuestas (retuits, respuestas a publicaciones, favoritos); y engagement (compromiso y vinculación con los seguidores). Estas métricas son de tipo cuantitativo, describiendo la actividad de la red social, y siendo el análisis de contenido de los comentarios un enfoque cualitativo.

Por último, según la tipología de red social se pueden lograr diferentes objetivos. Twitter es útil para crear compromiso por parte del consumidor y facilitar la propagación de las conversaciones acerca de la marca/producto/servicio. Facebook es un útil instrumento para la promoción, el desarrollo de comunidades online y la localización de diferentes segmentos de clientes (Lee y Cho, 2011; Castronovo y Huang, 2012; Järvinen et al., 2012). Las estaciones de esquí podrían optar para un contenido más caracterizado por la diversión y la promoción en su sentido más tradicional. 
Sin embargo, la literatura presenta una gran laguna respecto a estudios que analicen en detalle los indicadores proporcionados por las redes sociales referidos a las estaciones de esquí y menos aún a parques, aunque se reconozca su utilidad en el contexto actual (Quatman y Chelladurai, 2008; Gregory, Soberanis y Mejía, 2019).

\section{DISEÑO Y MÉTODO DE INVESTIGACIÓN}

En primer lugar, con la investigación diseñada se pretende conocer la valoración de los directivos y técnicos de las entidades que gestionan estaciones de esquí y parques. Para la realización de esta auditoría de marketing se recurre a la matriz importancia-valoración (IPA), una herramienta de gestión que adopta un enfoque de expectativas-desempeño para medir las percepciones de calidad y que ha sido ampliamente utilizada en la literatura de marketing (Matzler et al., 2003; Piñeiro et al., 2006). La técnica de recogida de datos es mediante encuesta estructurada a partir de la propuesta de Kotler y Dubois (1986), que identificaba seis dimensiones para la auditoría de marketing: entorno (cuestiones externas que afectan a la actividad como mercados, competencia, intermediarios entre otras); estrategia (objetivos y las grandes decisiones, como posicionamiento, desde la perspectiva de su adaptación al entorno y su evolución); modo de organización (capacidad para ejecutar y controlar la estrategia elegida); sistemas y procesos (adecuación de los sistemas de información, planificación y control así como los procesos de innovación); productividad (la rentabilidad de las diferentes acciones y el impacto de los presupuestos); y funciones (eficacia de los diferentes elementos del marketing-mix).

A partir de la propuesta de Kotler y Dubois (1986) y revisando la literatura se incorporaron hasta 83 ítems agrupados en las seis dimensiones mencionadas, se realizó un proceso de reducción para identificar los ítems más adecuados y en un número no excesivo. Estos ítems fueron seleccionados aplicando el método Delphi, por el que en dos oleadas fueron valorados por 4 expertos en marketing. Tras la primera valoración, cada experto tuvo acceso a la puntuación media de cada ítem (en una escala de 1 poco importante a 10 muy importante) antes de proceder a la segunda votación. Se seleccionaron los ítems de mayor puntuación media de importancia y menor desviación típica. Finalmente, el cuestionario quedó formado por 32 ítems repartidos en las seis dimensiones.

Se siguieron las características descritas por Kotler (1993): es independiente al realizarse por investigadores ajenos a la empresa; comprensiva, pues comprende todos los aspectos de marketing; sistemática, pues tiene un orden y estructura ordenada por dimensiones. Es importante mencionar que se trata de una auditoría independiente analizada por personal externo, pero la valoración es interna al ser los propios directivos quiénes evalúan sus propias organizaciones. Se siguieron las recomendaciones de Bell (1991) de realizar auditorías de gestión entre los altos estratos de la división organizacional y comenzar con el diagnóstico interno de los empleados.

La población objeto de estudio estaba constituida por PN de España, Italia, EEUU y México y por todas las estaciones de esquí españolas e italianas. Se envió a los directivos de los PN y de las estaciones, mediante correo electrónico, una dirección URL para que respondieran 
online el cuestionario autoadministrado (32 ítems con una escala de 1 a 10, para cada una de las dos dimensiones IPA: cómo estaba su PN o estación de esquí respecto a cada ítem, y qué importancia tenía para su desarrollo). Para la gestión de los cuestionarios se utilizó la plataforma Qualtrics. El trabajo de campo se llevó a cabo entre los meses de noviembre de 2017 y enero de 2018. Los detalles del trabajo de campo se sintetizan en la tabla 1.

En segundo lugar, la investigación perseguía conocer y analizar la actuación en redes sociales de estaciones de esquí y PN, para ello se recurrió a datos secundarios con las características que se muestran en la tabla 2.

Tabla 1. Resumen del trabajo de campo auditoría de marketing

\begin{tabular}{lll}
\hline Características & Estaciones esquí & Parques Nacionales/Naturales \\
\hline Universo & Estaciones alpinas: España e Italia. & España, EEUU, Italia y México \\
\hline Muestreo & Por conveniencia & Por conveniencia \\
\hline № respuestas & 29 & 41 \\
\hline Fecha & 10 Nov. 2017-10 Enero 2018 & 10 Nov. 2017-10 Enero 2018 \\
\hline № de Ítems & 32 (para las 6 dimensiones) & 32 (para las 6 dimensiones) \\
\hline Plataforma de entrevista & Qualtrics. Encuesta auto administrada & $\begin{array}{l}\text { Qualtrics. Encuesta auto } \\
\text { administrada }\end{array}$ \\
\hline
\end{tabular}

Elaboración propia.

Tabla 2. Características del estudio para el análisis de redes sociales

\begin{tabular}{|c|c|c|}
\hline Características & Estaciones esquí & Parques Nacionales/Naturales \\
\hline Muestra & $\begin{array}{l}\text { Estaciones españolas y } 20 \text { principales de } \\
\text { Italia }\end{array}$ & $\begin{array}{l}15 \text { PN de EEUU, España e Italia y } 14 \\
\text { de México }\end{array}$ \\
\hline Tipo de muestreo & Por conveniencia & Por conveniencia \\
\hline Fuente & $\begin{array}{l}\text { Para Facebook: LikeAlyzer } \\
\text { Para Twitter: Foller }\end{array}$ & $\begin{array}{l}\text { Para Facebook: LikeAlyzer } \\
\text { Para Twitter: Foller }\end{array}$ \\
\hline Fecha de consulta & 19 noviembre 2017 & 19 noviembre 2017 \\
\hline № de indicadores & $\begin{array}{l}\text { Para Facebook: } 15 \\
\text { Para Twitter: } 11\end{array}$ & $\begin{array}{l}\text { Para Facebook: } 15 \\
\text { Para Twitter: } 11\end{array}$ \\
\hline
\end{tabular}

Elaboración propia.

Los indicadores específicos de Twitter se muestran en la tabla 3 y los de Facebook en la tabla 4. 
Tabla 3. Indicadores de las páginas Twitter (Foller)

\begin{tabular}{ll}
\hline Indicador & Descripción \\
\hline Tweets & № total de tuits creados. \\
\hline Followers & № total de seguidores. \\
\hline Following & № total de seguidos. \\
\hline Followers per following & Ratio entre seguidores y seguidos. \\
\hline Listed & Total de las listas por las que se puede encontrar la cuenta. \\
\hline Replies & $\begin{array}{l}\text { Total de repuestas a los tuits de los seguidores. Este indicador se mide } \\
\text { considerando } 100 \text { tuits. }\end{array}$ \\
\hline Tweets with @mentions & № total de los tuits con menciones de la cuenta. Este indicador se mide \\
considerando 100 tuits.
\end{tabular}

Elaboración propia.

Tabla 4. Indicadores de las páginas Facebook (LikeAlyzer).

\begin{tabular}{|c|c|}
\hline Indicador & Descripción \\
\hline Frontpage & $\begin{array}{l}\text { \% de eficacia de la portada. Revisa la primera impresión visual al contar con foto } \\
\text { de portada. }\end{array}$ \\
\hline About & $\begin{array}{l}\text { \% de cantidad de información adicional que proporciona. Si incluye descripción } \\
\text { de la actividad, página web, medios de contacto o breve historia. }\end{array}$ \\
\hline Activity & $\begin{array}{l}\text { \% del nivel de actividad. Se trata de una medida general sobre el resto de } \\
\text { indicadores que se mencionan abajo. }\end{array}$ \\
\hline Response & $\begin{array}{l}\text { \% de respuesta de la página a los posts de sus seguidores. Si proporcionan } \\
\text { feedback a la actividad generada por los seguidores. }\end{array}$ \\
\hline Photos & $\%$ de fotos colgadas en el perfil. \\
\hline Videos & $\%$ de videos colgados en el perfil. \\
\hline Notes & $\%$ de notas colgadas en el perfil. Las notas se equiparan a las entradas de blog. \\
\hline $\begin{array}{l}\text { People talking about this } \\
\text { PTA }\end{array}$ & № de personas que han interactuado con la página en los últimos 7 días. \\
\hline Total Page likes & $\begin{array}{l}\text { Total de "me gusta" que ha recibido la página hasta la fecha. Se equipara al } \\
\text { número de seguidores. }\end{array}$ \\
\hline Engagement rate & $\begin{array}{l}\text { \% de compromiso con sus seguidores que tiene la página. Se calcula dividiendo } \\
\text { el número de "People talking about this" por el número total de "me gusta". }\end{array}$ \\
\hline
\end{tabular}




\begin{tabular}{ll}
\hline Posts per day & Media de post al día que crea la página. \\
\hline Average post length & Extensión media (en caracteres) de un post creado por la página. \\
\hline Events & № de eventos creados por los administradores. \\
\hline Pages liked & № de "me gusta" que ha dado la página a otras páginas. \\
\hline Native Facebook videos & № total de videos creados y colgados directamente. \\
\hline
\end{tabular}

Elaboración propia.

\section{ANÁLISIS Y DISCUSIÓN DE RESULTADOS}

En primer lugar, referidos a los PN, los aspectos más valorados según su importancia tienen que ver con el análisis del entorno (sobre todo buscar alternativas sostenibles, y adaptación a los cambios en la legislación), y con la estrategia de marketing como formular una clara misión o la importancia otorgada a la estrategia en Internet, y a gestionar la relación con los grupos de interés. También hay un ítem relacionado con la organización del parque (definir bien los puestos de trabajo relativos a las actividades de marketing con objetivos, responsabilidades y suficiente autoridad) y con funciones (disponer de un buen sistema de comunicación interna), considerados aspectos de importancia. Todos ellos superan una valoración media de 8 puntos.

En cuanto a lo que se daba menor importancia, las puntuaciones menores tenían que ver con funciones de marketing (realización de actividades de marketing directo y online; comunicación integrada de marketing); con aspectos relativos al control (tanto del coste de las actividades como de los objetivos que se persiguen); con la investigación (estudio de nuevos segmentos, analítica web); y con la formación y motivación de los empleados, como se muestra en la tabla 5. Para un análisis más detallado de todos los ítems y puntuaciones ver anexo 1.

Según los directivos y técnicos, los ítems con mayor importancia eran los que presentaban también una mejor valoración o adecuación, es decir lo que mejor se hace. Éstos hacían referencia, fundamentalmente, al entorno (conocer el impacto medioambiental, los cambios legislativos y los principales acontecimientos económicos); a la estrategia de relacionarse de forma adecuada con los grupos de interés; y a la definición de la misión. El tema de la organización también se realiza adecuadamente en cuanto a la definición correcta de los puestos de trabajo.

Por el contrario, las características peor valoradas (o principales debilidades), con puntuaciones menores fueron: seguimiento de las actividades de marketing, control de objetivos de marketing, comunicación integrada, utilizar analítica web, motivación de los empleados para la consecución de los objetivos de marketing, disponer de plan de marketing y revisar las relaciones con los operadores.

Entre los siete ítems con menos valoración de importancia, cinco de ellos son también los considerados como menos valorados en adecuación. 
Tabla 5. Características más y menos adecuadas e importantes según directivos de PN

\begin{tabular}{|c|c|c|c|}
\hline \multicolumn{2}{|c|}{ IMPORTANCIA } & \multicolumn{2}{|c|}{ ADECUACIÓN } \\
\hline MÁS & MENOS & MÁS & MENOS \\
\hline $\begin{array}{l}\text { Busca alternativas } \\
\text { sostenibles relacionadas } \\
\text { con el impacto ambiental }\end{array}$ & $\begin{array}{l}\text { Seguimiento del coste } \\
\text { de las actividades de } \\
\text { marketing }\end{array}$ & $\begin{array}{l}\text { Busca alternativas } \\
\text { sostenibles relacionadas } \\
\text { con el impacto ambiental }\end{array}$ & $\begin{array}{l}\text { Seguimiento del coste } \\
\text { de las actividades de } \\
\text { marketing }\end{array}$ \\
\hline $\begin{array}{l}\text { Puestos de trabajo } \\
\text { definidos con objetivos, } \\
\text { responsabilidades } \\
\text { y autoridad para } \\
\text { desarrollarlos }\end{array}$ & $\begin{array}{l}\text { Realización de actividades } \\
\text { de marketing directo, on } \\
\text { line y de base de datos }\end{array}$ & $\begin{array}{l}\text { La misión está formulada } \\
\text { con claridad, es factible } \\
\text { y conocida por todos } \\
\text { los miembros de la } \\
\text { organización }\end{array}$ & $\begin{array}{l}\text { Controla la consecución de } \\
\text { los objetivos de marketing } \\
\text { (offline y online) evalúa las } \\
\text { desviaciones }\end{array}$ \\
\hline $\begin{array}{l}\text { La misión está formulada } \\
\text { con claridad, es factible } \\
\text { y conocida por todos } \\
\text { los miembros de la } \\
\text { organización }\end{array}$ & $\begin{array}{l}\text { Comunicación integrada de } \\
\text { marketing }\end{array}$ & $\begin{array}{l}\text { Conoce y responde } \\
\text { rápidamente a los cambios } \\
\text { en la legislación que le } \\
\text { afectan }\end{array}$ & $\begin{array}{l}\text { Comunicación integrada de } \\
\text { marketing }\end{array}$ \\
\hline $\begin{array}{l}\text { La estrategia del parque } \\
\text { fomenta una buena } \\
\text { relación con sus grupos de } \\
\text { interés }\end{array}$ & $\begin{array}{l}\text { Controla la consecución de } \\
\text { los objetivos de marketing } \\
\text { (offline y online) evalúa las } \\
\text { desviaciones }\end{array}$ & $\begin{array}{l}\text { La estrategia del parque } \\
\text { fomenta una buena } \\
\text { relación con sus grupos de } \\
\text { interés }\end{array}$ & $\begin{array}{l}\text { Utiliza herramientas de } \\
\text { analítica web }\end{array}$ \\
\hline $\begin{array}{l}\text { Conoce y responde } \\
\text { rápidamente a los cambios } \\
\text { en la legislación que le } \\
\text { afectan }\end{array}$ & $\begin{array}{l}\text { Los empleados son } \\
\text { motivados para la } \\
\text { consecución de los } \\
\text { objetivos de marketing }\end{array}$ & $\begin{array}{l}\text { Tiene en cuenta los } \\
\text { principales acontecimientos } \\
\text { económicos de su entorno } \\
\text { para actuar }\end{array}$ & $\begin{array}{l}\text { Los empleados son } \\
\text { motivados para la } \\
\text { consecución de los } \\
\text { objetivos de marketing }\end{array}$ \\
\hline $\begin{array}{l}\text { Internet forma parte de la } \\
\text { estrategia de marketing }\end{array}$ & $\begin{array}{l}\text { Utiliza herramientas de } \\
\text { analítica web }\end{array}$ & $\begin{array}{l}\text { Internet forma parte de la } \\
\text { estrategia de marketing }\end{array}$ & $\begin{array}{l}\text { Dispone de un plan de } \\
\text { marketing coherente y } \\
\text { realizable }\end{array}$ \\
\hline $\begin{array}{l}\text { La estación dispone de un } \\
\text { sistema de comunicación } \\
\text { interna }\end{array}$ & $\begin{array}{l}\text { Busca nuevos segmentos } \\
\text { no satisfechos o nichos de } \\
\text { mercado }\end{array}$ & $\begin{array}{l}\text { Puestos de trabajo } \\
\text { definidos con objetivos, } \\
\text { responsabilidades } \\
\text { y autoridad para } \\
\text { desarrollarlos* } \\
\text { Internet forma parte de la } \\
\text { estrategia de marketing* }\end{array}$ & $\begin{array}{l}\text { Revisa periódicamente la } \\
\text { eficacia de los operadores } \\
\text { turísticos y comerciales con } \\
\text { los que opera }\end{array}$ \\
\hline
\end{tabular}

* Ítems con igual puntuación.

Elaboración propia.

En segundo lugar, referido únicamente a las estaciones de esquí, las características que se consideraron más importantes tienen que ver con el análisis del entorno ya que, en general, es importante conocer la opinión de los visitantes, investigar las necesidades de su público objetivo, y estar al tanto de acontecimientos económicos. También, hay una importancia de aspectos estratégicos (conoce su posición competitiva) y funcionales, sobre todo, de presencia web e Internet. En "importancia de organización de marketing" destaca una buena definición de los puestos de trabajo y la relación entre departamentos, como se comprueba en la tabla 6. 
En cuanto a lo que se daba menor importancia, las puntuaciones menores tenían que ver tanto con la productividad, estrategia y entorno de marketing: estudiar periódicamente el impacto social que sus servicios provocan entre las comunidades asentadas en la estación de esquí; disponer de un manual de identidad corporativa; o revisar el coste de las actividades periódicamente y se tomen las medidas oportunas; además de una misión bien definida; y la adopción del concepto de comunicación integrada de marketing.

Según los directivos, los ítems que presentaban una mejor valoración o adecuación coinciden bastante con los considerados más importantes. Es decir, lo que mejor se hace tiene que ver con entorno, organización, estrategia en Internet, y puestos de trabajo de la estación.

Por el contrario, las características peor valoradas (o principales debilidades), destacan el número de ítems relacionados con las funciones de marketing, sobre todo, con la comunicación (por ejemplo, que la comunicación se adapte al concepto de comunicación integrada de marketing, o el realizar actividades de marketing directo, online y de base de datos). Coinciden también con aquellos de menor importancia (revisar periódicamente la eficacia de los operadores turísticos).

Las puntuaciones de importancia son mayores que las de adecuación, con diferencias estadísticamente significativas (o cuasi-significativas), salvo para algunas excepciones (realizadas a través del test de Wilcoxon). Estas excepciones son las situaciones en las que no hay déficit, es decir, la consideración de adecuación está prácticamente al mismo nivel que la de importancia. Son seis ítems, relacionados tanto con la estrategia, entorno, funciones y productividad

Los mayores déficits son, en su mayoría, los relativos a las funciones: adaptar el concepto de comunicación integrada de marketing; realizar actividades de marketing directo, on line y de base de datos; o definir claramente la estrategia de calidad de su servicio. 
Tabla 6. Características más y menos adecuadas e importantes según directivos de estaciones de esquí

\begin{tabular}{|c|c|c|c|}
\hline \multicolumn{2}{|c|}{ IMPORTANCIA } & \multicolumn{2}{|c|}{ ADECUACIÓN } \\
\hline MÁS & MENOS & MÁS & MENOS \\
\hline $\begin{array}{l}\text { Internet forma parte } \\
\text { de la estrategia de } \\
\text { marketing }\end{array}$ & $\begin{array}{l}\text { Estudia periódicamente } \\
\text { el impacto social que sus } \\
\text { servicios provocan }\end{array}$ & $\begin{array}{l}\text { Internet forma parte } \\
\text { de la estrategia de } \\
\text { marketing }\end{array}$ & $\begin{array}{l}\text { La comunicación se } \\
\text { adapta al concepto de } \\
\text { comunicación integrada } \\
\text { de marketing }\end{array}$ \\
\hline $\begin{array}{l}\text { Conoce la opinión de sus } \\
\text { visitantes }\end{array}$ & $\begin{array}{l}\text { Dispone de un manual } \\
\text { de identidad corporativa }\end{array}$ & $\begin{array}{l}\text { Conoce y responde } \\
\text { rápidamente a los } \\
\text { cambios en la legislación } \\
\text { que le afectan }\end{array}$ & $\begin{array}{l}\text { Los empleados son } \\
\text { motivados para la } \\
\text { consecución de los } \\
\text { objetivos de marketing }\end{array}$ \\
\hline $\begin{array}{l}\text { Puestos de trabajo } \\
\text { definidos con objetivos, } \\
\text { responsabilidades } \\
\text { y autoridad para } \\
\text { desarrollarlos }\end{array}$ & $\begin{array}{l}\text { Dispone de información } \\
\text { sobre los objetivos, } \\
\text { estrategias, fortalezas } \\
\text { y debilidades de otras } \\
\text { estaciones }\end{array}$ & $\begin{array}{l}\text { Conoce su posición } \\
\text { competitiva, } \\
\text { sus amenazas y } \\
\text { oportunidades, para } \\
\text { definir objetivos }\end{array}$ & $\begin{array}{l}\text { Estudia periódicamente } \\
\text { el impacto social que sus } \\
\text { servicios provocan }\end{array}$ \\
\hline $\begin{array}{l}\text { Dispone de una página } \\
\text { web bien posicionada en } \\
\text { los buscadores }\end{array}$ & $\begin{array}{l}\text { El coste de las } \\
\text { actividades de } \\
\text { marketing es revisado } \\
\text { periódicamente y se } \\
\text { toman las medidas } \\
\text { oportunas }\end{array}$ & $\begin{array}{l}\text { Dispone de una página } \\
\text { web bien posicionada en } \\
\text { los buscadores }\end{array}$ & $\begin{array}{l}\text { Realiza actividades } \\
\text { de marketing directo, } \\
\text { marketing online y } \\
\text { marketing de base de } \\
\text { datos }\end{array}$ \\
\hline $\begin{array}{l}\text { Buena relación y } \\
\text { comunicación entre } \\
\text { el departamento de } \\
\text { marketing y los demás }\end{array}$ & $\begin{array}{l}\text { Revisa periódicamente } \\
\text { la eficacia de los } \\
\text { operadores turísticos y } \\
\text { comerciales con los que } \\
\text { opera }\end{array}$ & $\begin{array}{l}\text { Puestos de trabajo } \\
\text { definidos con objetivos, } \\
\text { responsabilidades } \\
\text { y autoridad para } \\
\text { desarrollarlos }\end{array}$ & $\begin{array}{l}\text { Revisa periódicamente } \\
\text { la eficacia de los } \\
\text { operadores turísticos y } \\
\text { comerciales con los que } \\
\text { opera }\end{array}$ \\
\hline $\begin{array}{l}\text { Investiga anualmente } \\
\text { las necesidades de su } \\
\text { público objetivo }\end{array}$ & $\begin{array}{l}\text { Misión formulada con } \\
\text { claridad }\end{array}$ & $\begin{array}{l}\text { Conoce la opinión de sus } \\
\text { visitantes }\end{array}$ & $\begin{array}{l}\text { Control de objetivos de } \\
\text { marketing }\end{array}$ \\
\hline $\begin{array}{l}\text { Conoce su posición } \\
\text { competitiva, } \\
\text { sus amenazas y } \\
\text { oportunidades, para } \\
\text { definir objetivos }\end{array}$ & $\begin{array}{l}\text { La comunicación se } \\
\text { adapta al concepto de } \\
\text { comunicación integrada } \\
\text { de marketing }\end{array}$ & $\begin{array}{l}\text { Personal de marketing } \\
\text { formado }\end{array}$ & $\begin{array}{l}\text { Dispone de un sistema } \\
\text { de comunicación interna }\end{array}$ \\
\hline
\end{tabular}

Elaboración propia.

\subsection{Comparación entre PN y estaciones de esquí}

Tras evaluar los resultados hallados según ambos sectores, se procede a comparar, en primer lugar, los ítems de importancia para conocer las diferencias entre los PN y las estaciones de esquí y, en segundo lugar, se hace lo mismo para los ítems de adecuación.

En cuanto a la valoración de la importancia, hay diferencias significativas en la mitad de los ítems (16 de 32). Solamente en dos ítems es mayor la importancia dada por los directivos de los PN, y son los relativos a impacto ambiental y definición de la misión. En los 14 
ítems restantes es mayor la importancia dada por los directivos de las estaciones de esquí pertenecientes a las dimensiones de estrategia de marketing, objetivos y actividades de marketing y comunicación. En particular: dispone de información sobre los objetivos, estrategias, fortalezas y debilidades de otras; tiene en cuenta los principales cambios tecnológicos de su entorno; dispone de un plan de marketing coherente y realizable; busca nuevos segmentos no satisfechos o nichos de mercado; la estrategia de la estación fomenta una buena relación con sus grupos de interés; hay buena relación y comunicación entre el departamento de marketing y los demás; los participantes en actividades de marketing están correctamente formados; los empleados son motivados para la consecución de los objetivos de marketing; controla la consecución de los objetivos de marketing (offline y online) evalúa las desviaciones; utiliza herramientas de analítica web; el coste de las actividades de marketing es revisado periódicamente y se toman las medidas oportunas; define y tiene claros sus objetivos de comunicación on y off line (publicidad y promoción); realiza actividades de marketing directo, marketing online y marketing de base de datos; la comunicación se adapta al concepto de comunicación integrada de marketing

En cuanto a la valoración de la adecuación las diferencias son aún mayores. Es muy diferente la adecuación de la gestión de marketing entre estaciones de esquí y parques puesto que hay diferencias significativas en 24 de 32 ítems. Únicamente en dos de los ítems es mayor la adecuación de los PN: relativos a impacto ambiental y definición de la misión, los mismos aspectos que los referidos a la importancia.

En 22 ítems es mayor la adecuación en estaciones de esquí que las de los parques, es decir, en todas las dimensiones identificadas desde la consideración de aspectos del entorno, como de estrategia, organización, productividad o funciones. El detalle de estos 22 ítems puede consultarse en el anexo 1.

Tras realizar un análisis pormenorizado de cada ítem, se ha procedido a comparar a nivel general la medida de adecuación, de importancia y de déficit. Para obtener una medida global de la importancia y también de adecuación tanto en estaciones de esquí como en parques, se procedió de la siguiente forma: se sumaron los ítems referidos a importancia, por un lado, y también los de adecuación, por otro, para obtener la puntuación media en cada una de las dos dimensiones. La fiabilidad de las escalas fue elevada, con un alfa de Cronbach de 0,958 para adecuación y de 0,961 para importancia.

Se realizaron análisis ANOVA para conocer la existencia o no de diferencias entre estaciones y parques tanto en importancia, como en adecuación y en déficits (o diferencia entre importancia y adecuación). En los tres análisis el test de Levene no fue significativo confirmando la existencia de homocedasticidad.

La adecuación total presentó diferencias estadísticamente significativas $(p=0,00)$, siendo mayor la de las estaciones de esquí que la de los PN.

Hubo diferencias estadísticamente cuasi-significativas $(p=0,09)$ entre estaciones de esquí y PN en cuanto a importancia total, siendo de nuevo mayor en las estaciones de esquí. 
En cuanto al déficit total, entendido como la diferencia entre importancia total menos adecuación total, hubo diferencias estadísticamente significativas $(p=0,001)$. Los PN tienen más déficit en la aplicación del marketing.

Tabla 7. ANOVA. Comparación PN-Estaciones de esquí

\begin{tabular}{|c|c|c|c|c|c|}
\hline & $\mathbf{N}$ & Media & $\begin{array}{l}\text { Desviación } \\
\text { típica }\end{array}$ & $\begin{array}{l}\text { Error } \\
\text { típico }\end{array}$ & Sig. \\
\hline \multicolumn{6}{|c|}{ DEFICIT } \\
\hline Estaciones de esquí & 27 & 9,4 & 48,86 & 9,40 & \multirow{3}{*}{0,00} \\
\hline PN & 39 & $-33,90$ & 52,52 & 8,41 & \\
\hline Total & 66 & $-16,17$ & 55,03 & 6,77 & \\
\hline \multicolumn{6}{|c|}{ ADECUACIÓN } \\
\hline Estaciones de esquí & 27 & 262,07 & 61,39 & 11,81 & \multirow{3}{*}{0,00} \\
\hline PN & 39 & 197,28 & 68,67 & 10,99 & \\
\hline Total & 66 & 223,79 & 72,77 & 8,957 & \\
\hline \multicolumn{6}{|c|}{ IMPORTANCIA } \\
\hline Estaciones de esquí & 27 & 252,63 & 48,13 & 9,26 & \multirow{3}{*}{0.09} \\
\hline PN & 39 & 231,18 & 53,19 & 8,52 & \\
\hline Total & 66 & 239,95 & 51,90 & 6,39 & \\
\hline
\end{tabular}

\subsection{Comparación de la actividad de estaciones de esquí y parques en Redes Sociales}

En el análisis de la actuación en redes sociales, respecto a Twitter lo primero que se comprueba es que todas las estaciones de esquí seleccionadas tienen cuenta en esta red social. Sin embargo, un total de 18 PN no cuentan con una página en Twitter. Tales PN pertenecen principalmente a España.

En la mayoría de los indicadores de Twitter no hubo diferencias significativas entre PN y estaciones de esquí (tabla 8), solamente hubo en 4 ítems. Hay que destacar que los PN superan a las estaciones de esquí en dos indicadores: followers y listed. Lo contrario ocurre en: tweets with links y tweets with media. 
Tabla 8. Valores medios de los PN y estaciones de esquí de los indicadores de Twitter. Fuente Foller. Nivel de significación para el test paramétrico de muestras independientes

\begin{tabular}{llrrr}
\hline Indicador & \multicolumn{1}{c}{ Descripción } & PN & Esquí & $\begin{array}{c}\text { Nivel de } \\
\text { significación }\end{array}$ \\
\hline Tweets & Número de tuits & 2690,24 & 3712,08 & 0,109 \\
\hline Followers & Número de seguidores & 49750,83 & 6174,88 & 0.035 \\
\hline Following & Número de seguidos & 280,88 & 272,16 & 0.902 \\
\hline Followers per following & Followers per following & 29,03 & 20,85 & 0.216 \\
\hline Listed & Listas seguidas & 388,19 & 96,51 & 0,002 \\
\hline Replies & Número de respuestas sobre 100 & 5,51 & 5,18 & 0.854 \\
\hline $\begin{array}{l}\text { Tweets with @ } \\
\text { mentions }\end{array}$ & Tuits con menciones sobre 100 & 38,73 & 38,31 & 0.945 \\
\hline Tweets with \#hashtags & Tuits con hashtags sobre 100 & & & 0.344 \\
\hline Retweets & Número de retweets sobre 100 & 37,71 & 43,31 & 0.566 \\
\hline Tweets with links & Tuit con links sobre 100 & 26,20 & 23,14 & 0.005 \\
\hline Tweets with media & Tuits con multimedia sobre 100 & 47,41 & 62,16 & 0.009 \\
\hline
\end{tabular}

Elaboración propia.

En cuanto a Facebook, las estaciones de esquí resultaron ser muchos más dinámicas que los PN prácticamente en todos los indicadores excepto en cuatro casos importantes: People talking about this, total Page likes, engagement rate, y average post length (tabla 9). Hay diferencias estadísticamente significativas en todos los indicadores relacionados con material o contenido subido a la página: Frontpage, About, Activity, Response, Photos, Videos y Notes siendo, en todas, mayor la actividad en las estaciones de esquí. Con diferencias cuasi-significativas $(0,084)$, también, superan a los PN en cuanto a la extensión media de un post creado por la página (average post length).

Sin embargo, también con diferencias estadísticamente significativas, es bastante superior el número de "me gusta" de los perfiles de Facebook de los PN así como el indicador referido al compromiso de sus seguidores (engagement rate). 
Tabla 9. Valores medios de los PN y estaciones de esquí de los indicadores de Facebook. Fuente LikeAlyzer. Nivel de significación para el test paramétrico de muestras independientes

\begin{tabular}{|c|c|c|c|c|}
\hline Indicador & Descripción & PN & Esquí & $\begin{array}{c}\text { Nivel de } \\
\text { significación }\end{array}$ \\
\hline Frontpage & Portada & 84,70 & 93,95 & 0,018 \\
\hline About & Información adicional & 65,91 & 93,21 & 0,000 \\
\hline Activity & $\begin{array}{l}\text { Porcentaje actividad de la } \\
\text { página }\end{array}$ & 41,81 & 87,25 & 0,000 \\
\hline Response & Respuestas & 28,21 & 85,21 & 0,000 \\
\hline Photos & Porcentaje fotos & 51,02 & 88,03 & 0,000 \\
\hline Videos & Porcentaje notas & 16,23 & 83,78 & 0,000 \\
\hline Notes & Porcentaje videos & 7,46 & 82,02 & 0,000 \\
\hline $\begin{array}{l}\text { People talking about } \\
\text { this }\end{array}$ & $\begin{array}{l}\text { Personas hablando de la } \\
\text { página }\end{array}$ & 3188,43 & 1610,30 & 0,105 \\
\hline Total Page likes & Total de "me gusta" recibidos & 116442,58 & 28993,00 & 0,008 \\
\hline Engagement rate & Implicación & 2,98 & 0,20 & 0,000 \\
\hline Posts per day & Post al día & 0,76 & 0,76 & 0,987 \\
\hline Average post length & Extensión media del post & 314,92 & 219,55 & 0,084 \\
\hline Events & Eventos creados & 3,02 & 3,03 & 1,000 \\
\hline Pages liked & Me gusta que da la página & 30,60 & 40,54 & 0,189 \\
\hline Native Facebook videos & Videos propios de la página & 6,12 & 6,73 & 0,686 \\
\hline
\end{tabular}

Se constata el mayor dinamismo de las estaciones de esquí en comparación con los parques, sobre todo en Facebook en donde tienen mucha más actividad (más fotos, videos, notas, o "me gusta") aunque no en post por día, ni en videos propios de la página, o personas que están hablando de la página.

\section{CONCLUSIONES}

El objetivo principal de este trabajo es conocer la gestión de marketing y el empleo de redes sociales en un análisis comparativo entre actividades o servicios relacionados con estaciones de esquí y parques naturales. El estudio presenta la novedad de realizar una auditoría de marketing comparativa entre las percepciones de directivos de estaciones de esquí y parques, así como de comparar la actividad en redes sociales de ambos sectores.

Los resultados alcanzados en la auditoría de marketing ponen de manifiesto que las estaciones de esquí, en general, realizan una gestión que consideran más adecuada, a la vez que otorgan una mayor importancia a las actividades de marketing. Ello puede deberse a su 
misión más enfocada a atraer visitantes, y a una mayor orientación a obtener beneficio. Sin embargo, tanto en las estaciones de esquí como en los PN, la dimensión que adquiere una mayor importancia es la del entorno, por lo que ambas comparten la necesidad del compromiso medioambiental y la convivencia con el entorno particular en las que actúan, en general la montaña. Precisamente, en el respecto ambiental y la definición de la misión la adecuación es mayor entre los PN y es más importante. Sin embargo, se han encontrado diferencias significativas en el déficit de la aplicación de marketing con la percepción de una gestión de marketing menos adecuada en los parques.

Así, los directivos y técnicos de los PN tienen una visión que difiere de los de las estaciones de esquí respecto su organización y ejecución desde una perspectiva de aplicación de marketing. Presentan una mayor sensibilidad al medio ambiente, a la necesidad de conservación y de un enfoque más sostenible, no perciben un contexto de competencia, ni la necesidad de incrementar visitantes como objetivo prioritario.

Estos resultados pueden servir como referente a modo de benchmarking, para que una estación de esquí o un parque de montaña pueda comprobar el grado de aplicación del marketing que desarrolla, y las fortalezas y debilidades que con respecto al resto del sector presenta.

A través del ejercicio de redes sociales se comprueba que la mayor orientación al mercado de las estaciones de esquí, no impide que los PN tengan un mayor número de seguidores y, sobre todo, un mayor compromiso de los participantes. A pesar de que muchos $\mathrm{PN}$ no cuentan con una página Twitter, aquellos que la tienen superan a las estaciones de esquí en seguidores y listas seguidas. En Facebook, también cuentan con mayor número de "me gusta" y de nivel de interacción (compromiso y gente hablando).

Efectivamente, las estaciones de esquí tienen un papel más activo en las redes sociales, puesto que generan más contenido, responden en mayor medida a los seguidores, y tienen mayor actividad diaria. De este modo, puede confirmarse que existe un compromiso con los $\mathrm{PN}$ en la sociedad, que se refleja en su número de seguidores y engagement, a pesar de que las estaciones de esquí están más enfocadas a este tipo de comunicación y tienen mayor actividad. En suma, los PN tienen mayor volumen en redes sociales (por los seguidores) pero las estaciones de esquí son mucho más dinámicas y activas en redes sociales.

Los resultados hallados pueden contribuir a una mejor gestión de marketing en estas entidades, identificando ámbitos importantes de mejora. El análisis de estas deficiencias puede contribuir a ser más eficientes, racionalizando y haciendo un seguimiento de los costes y mejorando las estrategias de desarrollo sostenible. Respecto a las redes sociales, cada vez más, las empresas utilizan instrumentos de analítica web para medir su desempeño, ya que esto tiene implicaciones positivas sobre los resultados de la empresa (Järvinen y Karjaluoto, 2015). A través de un buen análisis de las redes sociales, los gestores de estos servicios de montaña pueden monitorear a los distintos públicos y confeccionar estrategias de marketing hechas a medida para cada tipología de cliente/consumidor.

Tal como afirmaban Serbanica et al. (2015) y Morgan et al. (2002) son necesarias auditorias sobre la gestión del marketing desde todas las perspectivas para orientar y desarrollar un marketing dirigido a la sostenibilidad. 
A través del análisis de redes sociales, métricas como el número de interacciones, número de seguidores, compromiso, menciones, etc. son elementos de partida para analizar la efectividad de la actividad en redes sociales de los PN y las estaciones de esquí, y las nuevas tecnologías, de las que forman parte las redes sociales, hacen que los datos puedan ser recolectados, guardados, analizados y utilizados para redirigir las estrategias de marketing (Järvinen et al., 2012). Cuando los diferentes tipos de estrategias de marketing se unen a las redes sociales, los beneficios económicos son evidentes y medibles (Rapp et al., 2013).

Entre las limitaciones de la presente investigación, mencionar el tamaño de la muestra para la realización de la auditoría de marketing, que es reducido. No obstante, para este tipo de estudios consistentes en posicionar ítems, no son tan imprescindible grandes tamaños muestrales para tener un diagnóstico aceptable. Por otro lado, solamente se han considerado en el análisis dos redes sociales y determinados indicadores. Estas dos redes sociales son de las más utilizadas, pero hay otras que habrá de considerarse en un futuro. Además, las métricas utilizadas son descriptivas, lo que permite conocer cuáles tienen una mayor actividad o eficacia, pero no el desempeño a nivel cualitativo. Por último, se ha incluido diferentes países en cuanto a estaciones de esquí y áreas naturales protegidas.

Como futuras líneas de investigación sería interesante ampliar el número de países u otros servicios relacionados con la montaña, así como desarrollar una comparación según otras vías de comunicación online de las estaciones de esquí como, por ejemplo, sus páginas webs. Así mismo, sería pertinente un análisis cualitativo de los comentarios y las publicaciones generadas por los perfiles de sus redes sociales para profundizar en la conexión emocional, si se generan más respuestas positivas que negativas, y cuáles son los principales temas o aspectos sobre los que publican.

\section{REFERENCIAS BIBLIOGRÁFICAS}

Alalwan, A. A., Rana, N. P., Dwivedi, Y. K., \& Algharabat, R. (2017). Social media in marketing: A review and analysis of the existing literature. Telematics and Informatics, 34(7), 1177-1190.

Alarcón, C. N., Sepúlveda, A. U., Valenzuela-Fernández, L., \& Gil-Lafuente, J. (2018). Systematic mapping on social media and its relation to business. European Research on Management and Business Economics, 24(2), 104-113.

Algharabat, R., Alalwan, A. A., Rana, N. P., \& Dwivedi, Y. K. (2017). Three dimensional product presentation quality antecedents and their consequences for online retailers: The moderating role of virtual product experience. Journal of Retailing and Consumer Services, 36, 203-217.

Algharabat, R., Rana, N. P., Dwivedi, Y. K., Alalwan, A. A., \& Qasem, Z. (2018). The effect of telepresence, social presence and involvement on consumer brand engagement: An empirical study of non-profit organizations. Journal of Retailing and Consumer Services, 40, 139-149.

Andzulis, J. M., Panagopoulos, N. G., \& Rapp, A. (2012). A review of social media and implications for the sales process. Journal of Personal Selling \& Sales Management, 32(3), 305-316. 
Baena, V. (2016). Online and mobile marketing strategies as drivers of brand love in sports teams: findings from Real Madrid. International Journal of Sports Marketing and Sponsorship, 17(3), 202-218.

Baker, W. (1990) 'Market networks and corporate behavior', American Journal of Sociology, Vol. 96, No. 3, pp.589-625.

Bell, M. (1991). Mercadotecnia. México: Editorial Continental.

Benson, C., Watson, P., Taylor, G., Cook, P., \& Hollenhorst, S. (2013). Who visits a national park and what do they get out of it?: A joint visitor cluster analysis and travel cost model for Yellowstone National Park. Environmental management, 52(4), 917-928.

Bulearca, M., \& Bulearca, S. (2010). Twitter: a viable marketing tool for SMEs? Global Business and Management Research, 2(4), 296.

Canadian Heritage Canada Parks. (2017). State of Parks reports for national parks. Obtenido de https://www.pc.gc.ca/en/agence-agency/bib-lib/rapports-reports/ tarification-fees/2018-2019

Castronovo, C., \& Huang, L. (2012). Social media in an alternative marketing communication model. Journal of Marketing Development and Competitiveness, 6(1), 117-134.

Cheng, Ch., 2010. A new marketing audit value model based on fuzzy theory and main component analysis method. In: IEEE, ICIME 2010, 2nd IEEE International Conference on Information Management and Engineering. Chengdu, China, 16-18 April 2010. New York: IEEE.

Coelho, R. L. F., Oliveira, D. S. D., \& Almeida, M. I. S. D. (2016). Does social media matter for post typology? Impact of post content on Facebook and Instagram metrics. Online Information Review, 40(4), 458-471.

Corbera, E., Kosoy, N., \& Tuna, M. M. (2007). Equity implications of marketing ecosystem services in protected areas and rural communities: Case studies from Meso-America. Global Environmental Change, 17(3-4), 365-380.

da Gama, A. P. (2012). Marketing audits: The forgotten side of management? Journal of Targeting, Measurement and Analysis for Marketing, 20(3-4), 212-222.

Daries-Ramon, N., Cristóbal-Fransi, E., Martin-Fuentes, E., \& Marine-Roig, E. (2016). Adopción del comercio electrónico en el turismo de nieve y montaña: análisis de la presencia web de las estaciones de esquí a través del Modelo eMICA. Cuadernos de Turismo, 37, 113-134.

Doyle, S. (2007). The role of social networks in marketing. Journal of Database Marketing \& Customer Strategy Management, 15(1), 60-64.

Dickson, T. J., \& Faulks, P. (2007). Exploring overseas snowsport participation by Australian skiers and snowboarders. Tourism Review, 62(3/4), 7-14.

Eggers, F., Hatak, I., Kraus, S., \& Niemand, T. (2017). Technologies that support marketing and market development in SMEs-Evidence from social networks. Journal of Small Business Management, 55(2), 270-302.

Filo, K., Lock, D., \& Karg, A. (2015). Sport and social media research: A review. Sport Management Review, 18(2), 166-181.

Gecti, F., \& Dastan, I. (2013). The impact of social media-focused information \& communication technologies on business performance via mediating mechanisms: An exploratory 
study on communication and advertising agencies in Turkey. International Journal of Business and Management, 8(7), 106.

Gilmore, A., \& Simmons, G. (2007). Integrating sustainable tourism and marketing management: can National Parks provide the framework for strategic change? Strategic change, 16(5), 191-200.

Goeldner, C. R., \& Ritchie, J. B. (2007). Tourism principles, practices, philosophies. John Wiley $\&$ Sons.

Godfrey, K. B. (1999). Attributes of destination choice: British skiing in Canada. Journal of Vacation Marketing, 5(1), 18-30.

Gregory, R. B., Soberanis, F. E., \& Mejía, J. A. M. (2019). La capacidad de carga psicosocial del turista: instrumento de medición para el desarrollo sostenible en la turistificación de los cenotes. Cuadernos de Turismo, (43), 169-186.

Hall, J., O'Mahony, B., \& Gayler, J. (2017). Modelling the relationship between attribute satisfaction, overall satisfaction, and behavioural intentions in Australian ski resorts. Journal of Travel \& Tourism Marketing, 34(6), 764-778.

Hudson, S., Roth, M. S., Madden, T. J., \& Hudson, R. (2015). The effects of social media on emotions, brand relationship quality, and word of mouth: An empirical study of music festival attendees. Tourism Management, 47, 68-76.

Hudson, S., \& Shephard, G. W. (1998). Measuring service quality at tourist destinations: An application of importance-performance analysis to an alpine ski resort. Journal of Travel \& Tourism Marketing, 7(3), 61-77.

Hung, K. H., \& Li, S. Y. (2007). The influence of eWOM on virtual consumer communities: Social capital, consumer learning, and behavioural outcomes. Journal of Advertising Research, 47(4), 485-495.

Hunt, S. D. (1983). General theories and the fundamental explananda of marketing. The Journal of Marketing, 9-17.

Hwa-Ryong, K., \& Sung-Kyeom, K. (2010). Importance-Satisfaction Analysis of Attribute Assessment for Ski Resort. Korean Journal of Sports Science, 19(4), 715-731.

loakimidis, M. (2010). Online marketing of professional sports clubs: Engaging fans on a new playing field. International Journal of Sports Marketing and Sponsorship, 11(4), 2-13.

Ismert, M., \& Petrick, J. F. (2004). Indicators and standards of quality related to seasonal employment in the ski industry. Journal of Travel Research, 43(1), 46-56.

IUCN (2016). Protected Planet Report. Cambridge UK.

Jamrozy, U. (2008). Sustainable tourism development: ingenuity in marketing strategy. WIT Transactions on Ecology and the Environment, 115.

Järvinen, J., \& Karjaluoto, H. (2015). The use of Web analytics for digital marketing performance measurement. Industrial Marketing Management, 50 (October), 117- 127. doi:10.1016/j.indmarman.2015.04.009

Järvinen, J., Tollinen, A., Karjaluoto, H., \& Jayawardhena, C. (2012). Digital and social media marketing usage in b2b industrial section. Marketing Management Journal, 22(2).

Kang, J. H., Bagozzi, R. P., \& Oh, J. (2011). Emotions as antecedents of participant sport consumption decisions: a model integrating emotive, self-based, and utilitarian evaluations. Journal of sport Management, 25(4), 314-325. 
Kietzmann, J. H., Hermkens, K., McCarthy, I. P., \& Silvestre, B. S. (2011). Social media? Get serious! Understanding the functional building blocks of social media. Business Horizons, 54(3), 241-251.

Kihima, B. O. (2014). Unlocking the Kenyan Tourism Potential Through Park Branding Exercise. Tourism Recreation Research, 39(1), 51-64.

Kim, W. G., Lim, H., \& Brymer, R. A. (2015). The effectiveness of managing social media on hotel performance. International Journal of Hospitality Management, 44, 165-171.

Kim, D. H., Spiller, L., \& Hettche, M. (2015). Analyzing media types and content orientations in Facebook for global brands. Journal of Research in Interactive Marketing, 9(1), 4-30.

Konu, H., Laukkanen, T., \& Komppula, R. (2011). Using ski destination choice criteria to segment Finnish ski resort customers. Tourism Management, 32(5), 1096-1105.

Kotler, P. \& Dubois, B. (2003). Marketing management. Publi Union.

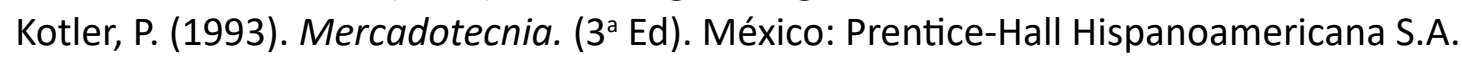

Kotler, P, \& Keller, K. (2009). Marketing Management. New Jersey: Pearson International Edition.

Kruger, M., Viljoen, A., \& Saayman, M. (2017). Who visits the Kruger National Park, and why? identifying target markets. Journal of Travel \& Tourism Marketing, 34(3), 312-340.

Lamb, C. W., \& Crompton, J. L. (2017). Analyzing marketing performance. In Marketing the Public Sector (pp. 173-184). Routledge.

Lai, P. H., Sorice, M. G., Nepal, S. K., \& Cheng, C. K. (2009). Integrating social marketing into sustainable resource management at Padre Island National Seashore: an attitude-based segmentation approach. Environmental Management, 43(6), 985.

Layton, R. A., \& Grossbart, S. (2006). Macromarkeing: Past, present, and possible future. Journal of Macromarketing, 26(2), 193-213.

Lee, S., \& Cho, M. (2011). Social media use in a mobile broadband environment: Examination of determinants of Twitter and Facebook use. International Journal of Mobile Marketing, 6(2), 71-87.

Luarn, P., Lin, Y. F., \& Chiu, Y. P. (2015). Influence of Facebook brand-page posts on online engagement. Online Information Review, 39(4), 505-519.

McDonald, M., \& Wilson, H. (2016). Marketing Plans: How to prepare them, how to profit from them. John Wiley \& Sons.

MAPAMA (30 de 12 de 2017). Red de Parques Nacionales. Obtenido de http://www.mapama. gob.es/es/red-parques-nacionales/la-red/historia.aspx

Matzler, K. \& Siller, H.J. (2003). Linking travel motivations with perceptions of destinations: the case of youth travelers in Alpine summer and winter tourism. Tourism Review, 58 (4), 6-11.

Miragaia, D., Conde, D., \& Soares, J. (2016). Measuring Service Quality of Ski Resorts: An Approach to Identify the Consumer Profile. The Open Sports Sciences Journal, 9 (1).

Mokwa, M. (1986) The strategic marketing audit: An adoption/utilization perspective. The Journal of Business Strategy 6 (4), 88-95.

Morgan, N. A., Clark, B. H., \& Gooner, R. (2002). Marketing productivity, marketing audits, and systems for marketing performance assessment: integrating multiple perspectives. Journal of Business Research, 55(5), 363-375. 
Olcina Cantos, J. (2012). Turismo y cambio climático: una actividad vulnerable que debe adaptarse. Investigaciones Turísticas, 4, 1-31.

Paniagua, J., \& Sapena, J. (2014). Business performance and social media: Love or hate? Business Horizons, 57(6), 719-728.

Pearce, D. G., \& Schänzel, H. A. (2013). Destination management: The tourists' perspective. Journal of Destination Marketing \& Management, 2(3), 137-145.

Pérez Hernández, I., \& Ramírez Pérez, J. F. (2013). Aportaciones al desarrollo ecoturístico sostenible, a partir de las relaciones intersectoriales en áreas protegidas. Experiencia Parque Nacional Viñales, Cuba. Investigaciones Turísticas, (6), 71-94.

Quatman, C., \& Chelladurai, P. (2008). Social network theory and analysis: A complementary lens for inquiry. Journal of Sport Management, 22(3), 338-360.

Rapp, A., Beitelspacher, L. S., Grewal, D., \& Hughes, D. E. (2013). Understanding social media effects across seller, retailer, and consumer interactions. Journal of the Academy of Marketing Science, 41(5), 547-566.

Reinius, S. W., \& Fredman, P. (2007). Protected areas as attractions. Annals of tourism research, 34(4), 839-854.

Rothschild, P. C. (2011). Social media use in sports and entertainment venues. International Journal of Event and Festival Management, 2(2), 139e150.

Sabate, F., Berbegal-Mirabent, J., Cañabate, A., \& Lebherz, P. R. (2014). Factors influencing popularity of branded content in Facebook fan pages. European Management Journal, 32(6), 1001-1011.

SEMARTAT-CONANP (2014). Programa Nacional de Areas Naturales Protegidas 2014-2018. México D.F.

Serbanica, D., Radulescu, V., \& Cruceru, A. F. (2015). The Role of Marketing Audit in Evaluation Sustainable Marketing Performance in Romanian Organizations. Amfiteatru Economic, 17(40), 1011.

Sharpley, R., \& Pearce, T. (2007). Tourism, marketing and sustainable development in the English national parks: The role of national park authorities. Journal of Sustainable Tourism, 15(5), 557-573.

Taghian, M., \& Shaw, R. N. (2008). The marketing audit and organizational performance: an empirical profiling. Journal of Marketing Theory and Practice, 16(4), 341-350.

Taylor, P. A., \& Grandjean, B. D. (2009, January). Visitor satisfaction and support for park fees: Examining the effects of front country, backcountry, and information in Rocky mountain national park. In The George Wright Forum (Vol. 26, No. 2, pp. 12-21). George Wright Society.

Tjørve, E., Lien, G., \& Flognfeldt, T. (2015). Properties of first-time vs. repeat visitors: lessons for marketing Norwegian ski resorts. Current Issues in Tourism, 1-25.

Weller, K. (2015). Accepting the challenges of social media research. Online Information Review, 39(3), 281-289.

Wilson, A. (2005). Marketing Audit Handbook. Kogan Page Publishers.

Zaglia, M. E. (2013). Brand communities embedded in social networks. Journal of Business Research, 66(2), 216-223.

Zhu, Y. Q., \& Chen, H. G. (2015). Social media and human need satisfaction: Implications for social media marketing. Business Horizons, 58(3), 335-345. 
ANEXO 1. PUNTUACIONES DE IMPORTANCIA Y DE ADECUACIÓN. DIFERENCIAS ENTRE IMPORTANCIA Y ADECUACIÓN Y NIVEL DE SIGNIFICACIÓN (ANOVA) PARA LAS DIFERENCIAS ENTRE IMPORTANCIA Y ADECUACIÓN.

\begin{tabular}{|c|c|c|c|c|c|c|c|c|}
\hline \multirow[t]{2}{*}{ Estadísticos de grupo } & \multicolumn{6}{|c|}{ ADECUACIÓN } & \multicolumn{2}{|c|}{ IMPORTANCIA } \\
\hline & $\begin{array}{l}\text { Tipo de } \\
\text { entidad }\end{array}$ & $\mathrm{N}$ & Media & $\begin{array}{l}\text { Desviación } \\
\text { típ. }\end{array}$ & Sig & Media & $\begin{array}{l}\text { Desviación } \\
\text { típ. }\end{array}$ & Sig \\
\hline \multirow{2}{*}{$\begin{array}{l}\text { Tiene en cuenta los principales acontecimientos económicos de su } \\
\text { entorno para actuar }\end{array}$} & Esquí & 29 & 7,41 & 1,78 & 0,29 & 7,72 & 1,73 & 0,86 \\
\hline & PN & 39 & 6,97 & 1,63 & & 7,79 & 1,44 & \\
\hline \multirow[t]{2}{*}{ Busca alternativas sostenibles relacionadas con el impacto ambiental } & Esquí & 29 & 7,14 & 2,28 & 0,01 & 7,59 & 2,06 & 0,00 \\
\hline & PN & 39 & 8,36 & 1,01 & & 9,23 & 0,81 & \\
\hline \multirow[t]{2}{*}{ Investiga anualmente las necesidades de su público objetivo } & Esquí & 29 & 7,03 & 2,20 & 0,01 & 8,31 & 2,32 & 0,15 \\
\hline & PN & 39 & 5,54 & 2,46 & & 7,59 & 1,77 & \\
\hline \multirow[t]{2}{*}{ Conoce la opinión de sus visitantes (reales y potenciales) } & Esquí & 29 & 7,45 & 1,68 & 0,02 & 8,52 & 1,81 & 0,19 \\
\hline & PN & 39 & 6,33 & 1,98 & & 7,97 & 1,55 & \\
\hline \multirow{2}{*}{$\begin{array}{l}\text { Dispone de información sobre los objetivos, estrategias, fortalezas y } \\
\text { debilidades de otras estaciones }\end{array}$} & Esquí & 29 & 6,55 & 2,06 & 0,01 & 7,31 & 1,98 & 0,04 \\
\hline & PN & 39 & 5,18 & 2,27 & & 6,26 & 2,04 & \\
\hline \multirow{2}{*}{$\begin{array}{l}\text { Conoce y responde rápidamente a los cambios en la legislación que le } \\
\text { afectan }\end{array}$} & Esquí & 29 & 8,00 & 1,46 & 0,70 & 8,28 & 1,53 & 0,44 \\
\hline & PN & 39 & 7,82 & 2,13 & & 8,62 & 1,97 & \\
\hline \multirow[t]{2}{*}{ Tiene en cuenta los principales cambios tecnológicos de su entorno } & Esquí & 29 & 7,07 & 1,53 & 0,01 & 7,86 & 1,62 & 0,03 \\
\hline & PN & 39 & 5,90 & 1,96 & & 6,85 & 1,97 & \\
\hline \multirow{2}{*}{$\begin{array}{l}\text { La misión está formulada con claridad, es factible y conocida por todos } \\
\text { los miembros de la organización }\end{array}$} & Esquí & 29 & 6,86 & 1,96 & 0,01 & 7,48 & 2,25 & 0,01 \\
\hline & PN & 39 & 8,10 & 1,60 & & 8,85 & 1,97 & \\
\hline \multirow[t]{2}{*}{ Dispone de un plan de marketing coherente y realizable } & Esquí & 29 & 7,00 & 2,25 & 0,00 & 8,03 & 2,37 & 0,02 \\
\hline & PN & 39 & 3,46 & 2,64 & & 6,69 & 2,24 & \\
\hline
\end{tabular}




\begin{tabular}{|c|c|c|c|c|c|c|c|c|}
\hline La estrategia de marketing tiene en cuenta la carga turística adecuada & Esquí & 29 & 7,00 & 2,14 & 0,00 & 7,69 & 2,17 & 0,64 \\
\hline & PN & 39 & 4,56 & 3,02 & & 7,41 & 2,55 & \\
\hline \multirow[t]{2}{*}{ Busca nuevos segmentos no satisfechos o nichos de mercado } & Esquí & 29 & 6,62 & 2,26 & 0,00 & 7,69 & 2,32 & 0,03 \\
\hline & PN & 39 & 4,54 & 2,97 & & 6,23 & 3,03 & \\
\hline \multirow[t]{2}{*}{ Dispone de un manual de identidad corporativa } & Esquí & 29 & 6,45 & 3,02 & 0,99 & 7,28 & 2,66 & 0,29 \\
\hline & PN & 39 & 6,44 & 3,15 & & 7,92 & 2,29 & \\
\hline \multirow{2}{*}{$\begin{array}{l}\text { La estrategia de la estación fomenta una buena relación con sus grupos } \\
\text { de interés }\end{array}$} & Esquí & 29 & 7,07 & 1,77 & 0,31 & 7,93 & 1,91 & 0,03 \\
\hline & PN & 39 & 7,51 & 1,76 & & 8,82 & 1,32 & \\
\hline \multirow[t]{2}{*}{ Internet forma parte de la estrategia de marketing } & Esquí & 29 & 8,31 & 1,65 & 0,01 & 8,72 & 1,33 & 0,25 \\
\hline & PN & 39 & 6,87 & 2,70 & & 8,23 & 1,99 & \\
\hline \multirow{2}{*}{$\begin{array}{l}\text { Conoce su posición competitiva, sus amenazas y oportunidades, para } \\
\text { definir objetivos }\end{array}$} & Esquí & 29 & 7,66 & 1,59 & 0,00 & 8,31 & 1,39 & 0,24 \\
\hline & PN & 39 & 6,15 & 2,65 & & 7,74 & 2,28 & \\
\hline \multirow{2}{*}{$\begin{array}{l}\text { Puestos de trabajo definidos con objetivos, responsabilidades y autoridad } \\
\text { para desarrollarlos }\end{array}$} & Esquí & 27 & 7,59 & 1,69 & 0,13 & 8,41 & 1,58 & 0,23 \\
\hline & PN & 41 & 6,80 & 2,51 & & 8,88 & 1,54 & \\
\hline \multirow{2}{*}{$\begin{array}{l}\text { Buena relación y comunicación entre el departamento de marketing y los } \\
\text { demás }\end{array}$} & Esquí & 27 & 7,37 & 2,10 & 0,00 & 8,37 & 1,67 & 0,00 \\
\hline & PN & 41 & 4,95 & 3,47 & & 6,54 & 3,39 & \\
\hline \multirow[t]{2}{*}{ Participantes en actividades de marketing están correctamente formados } & Esquí & 27 & 7,44 & 1,67 & 0,00 & 8,30 & 1,44 & 0,00 \\
\hline & PN & 41 & 4,07 & 3,12 & & 6,56 & 3,22 & \\
\hline \multirow{2}{*}{$\begin{array}{l}\text { Los empleados son motivados para la consecución de los objetivos de } \\
\text { marketing }\end{array}$} & Esquí & 27 & 6,00 & 2,25 & 0,00 & 7,67 & 2,15 & 0,02 \\
\hline & PN & 41 & 3,44 & 2,99 & & 5,95 & 3,18 & \\
\hline $\begin{array}{l}\text { Controla la consecución de los objetivos de marketing (offline y online) } \\
\text { evalua las desviaciones }\end{array}$ & Esquí & 27 & 6,22 & 2,46 & 0,00 & 7,59 & 2,34 & 0,01 \\
\hline
\end{tabular}




\begin{tabular}{|c|c|c|c|c|c|c|c|c|}
\hline & PN & 41 & 2,93 & 2,84 & & 5,61 & 3,29 & \\
\hline \multirow[t]{2}{*}{ Utiliza herramientas de analítica web } & Esquí & 27 & 7,00 & 2,08 & 0,00 & 7,70 & 2,15 & 0,02 \\
\hline & PN & 41 & 3,32 & 2,93 & & 6,22 & 2,79 & \\
\hline \multirow[t]{2}{*}{ Estudia periódicamente el impacto social que sus servicios provocan } & Esquí & 27 & 6,07 & 2,75 & 0,01 & 7,04 & 2,39 & 0,57 \\
\hline & PN & 41 & 4,39 & 2,63 & & 7,37 & 2,25 & \\
\hline \multirow{2}{*}{$\begin{array}{l}\text { Comparte programas a través de acuerdos de colaboración con otras } \\
\text { instituciones }\end{array}$} & Esquí & 27 & 6,96 & 1,99 & 0,07 & 7,78 & 1,74 & 0,38 \\
\hline & PN & 41 & 5,85 & 2,74 & & 7,32 & 2,58 & \\
\hline \multirow{2}{*}{$\begin{array}{l}\text { El coste de las actividades de marketing es revisado periódicamente y se } \\
\text { toman las medidas oportunas }\end{array}$} & Esquí & 27 & 6,96 & 2,30 & 0,00 & 7,41 & 2,61 & 0,01 \\
\hline & PN & 41 & 2,73 & 3,07 & & 5,34 & 3,32 & \\
\hline \multirow[t]{2}{*}{ Dispone de una página web bien posicionada en los buscadores } & Esquí & 27 & 7,63 & 1,60 & 0,00 & 8,37 & 1,67 & 0,35 \\
\hline & $\mathrm{PN}$ & 41 & 5,93 & 2,70 & & 7,88 & 2,37 & \\
\hline \multirow[t]{2}{*}{ Claramente definida su estrategia de calidad de su servicio } & Esquí & 27 & 7,11 & 1,87 & 0,02 & 8,30 & 1,59 & 0,31 \\
\hline & $\mathrm{PN}$ & 41 & 5,68 & 3,05 & & 7,73 & 2,56 & \\
\hline \multirow[t]{2}{*}{ La estación dispone de un sistema de comunicación interna } & Esquí & 27 & 6,44 & 1,99 & 0,58 & 7,89 & 1,70 & 0,79 \\
\hline & PN & 41 & 6,80 & 2,98 & & 8,02 & 2,30 & \\
\hline \multirow{2}{*}{$\begin{array}{l}\text { Define y tiene claros sus objetivos de comunicación on y off line } \\
\text { (publicidad y promoción) }\end{array}$} & Esquí & 27 & 6,85 & 2,20 & 0,00 & 8,22 & 2,04 & 0,04 \\
\hline & PN & 41 & 4,98 & 2,66 & & 7,00 & 2,67 & \\
\hline \multirow{2}{*}{$\begin{array}{l}\text { Los servicios presentan algún valor añadido que lo diferencie de las } \\
\text { demás estaciones }\end{array}$} & Esquí & 27 & 7,00 & 2,50 & 0,41 & 7,70 & 2,43 & 0,69 \\
\hline & $\mathrm{PN}$ & 41 & 6,49 & 2,45 & & 7,46 & 2,35 & \\
\hline \multirow{2}{*}{$\begin{array}{l}\text { Revisa periódicamente la eficacia de los operadores turísticos y } \\
\text { comerciales con los que opera }\end{array}$} & Esquí & 27 & 6,19 & 2,62 & 0,00 & 7,41 & 2,66 & 0,20 \\
\hline & $\mathrm{PN}$ & 41 & 3,73 & 3,27 & & 6,46 & 3,12 & \\
\hline
\end{tabular}




\begin{tabular}{|c|c|c|c|c|c|c|c|c|}
\hline $\begin{array}{l}\text { Realiza actividades de marketing directo, marketing online y marketing } \\
\text { de base de datos }\end{array}$ & Esquí & 27 & 6,15 & 2,45 & 0,00 & 7,70 & 2,16 & 0,00 \\
\hline & PN & 41 & 3,12 & 2,89 & & 5,44 & 3,33 & \\
\hline \multirow{2}{*}{$\begin{array}{l}\text { La comunicación se adapta al concepto de comunicación integrada de } \\
\text { marketing }\end{array}$} & Esquí & 27 & 5,96 & 2,52 & 0,00 & 7,56 & 2,69 & 0,01 \\
\hline & PN & 41 & 2,95 & 2,95 & & 5,56 & 3,16 & \\
\hline
\end{tabular}

\begin{tabular}{|c|c|c|c|c|c|c|c|c|c|}
\hline & & \multirow{2}{*}{$\mathbf{N}$} & \multirow{2}{*}{ Media } & \multirow{2}{*}{$\begin{array}{l}\text { Desviación } \\
\text { típica }\end{array}$} & \multirow{2}{*}{$\begin{array}{l}\text { Error } \\
\text { típico }\end{array}$} & \multicolumn{2}{|c|}{$\begin{array}{l}\text { Intervalo de confianza } \\
\text { para la media al } 95 \%\end{array}$} & \multirow{2}{*}{ Mínimo } & \multirow{2}{*}{ Máximo } \\
\hline & & & & & & Límite inferior & Límite superior & & \\
\hline \multirow[t]{3}{*}{ Nivel de adecuación } & Estaciones de esquí & 27 & 262,07 & 61,40 & 11,82 & 237,79 & 286,36 & 98 & 349 \\
\hline & PN & 39 & 197,28 & 68,67 & 11,00 & 175,02 & 219,54 & 72 & 382 \\
\hline & Total & 66 & 223,79 & 72,77 & 8,96 & 205,90 & 241,68 & 72 & 382 \\
\hline \multirow[t]{3}{*}{ Nivel de importancia } & Estaciones de esquí & 27 & 252,63 & 48,13 & 9,26 & 233,59 & 271,67 & 98 & 315 \\
\hline & PN & 39 & 231,18 & 53,19 & 8,52 & 213,94 & 248,42 & 118 & 320 \\
\hline & Total & 66 & 239,95 & 51,90 & 6,39 & 227,20 & 252,71 & 98 & 320 \\
\hline \multirow[t]{3}{*}{ Déficits } & Estaciones de esquí & 27 & 9,44 & 48,86 & 9,40 & $-9,88$ & 28,77 & -123 & 97 \\
\hline & PN & 39 & $-33,90$ & 52,53 & 8,41 & $-50,92$ & $-16,87$ & -181 & 77 \\
\hline & Total & 66 & $-16,17$ & 55,04 & 6,77 & $-29,70$ & $-2,64$ & -181 & 97 \\
\hline
\end{tabular}

